\title{
MRI-Detected Cranial Nerve Involvement in Nasopharyngeal Carcinoma
}

\author{
Li Li, Wenxin Yuan, Lizhi Liu and Chunyan Cui \\ Cancer Center, Sun Yat-Sen University
}

China

\section{Introduction}

The incidence of cranial nerve (CN) palsy is not uncommon in patients with untreated nasopharyngeal cancer (NPC). In the 7th edition of the American Joint Committee on Cancer (AJCC) Staging Manual, the AJCC recommended that $\mathrm{CN}$ involvement be assessed by neurological evaluation rather than by cross-sectional imaging (computed tomography [CT] and magnetic resonance imaging [MRI]), because $\mathrm{CN}$ involvement is considered a poor prognostic indicator in NPC patients, those with CN involvement are staged as T4.

However, evaluation of $\mathrm{CN}$ palsy by clinical symptoms and physical examination has limitations. First, the accuracy of the neurological examination depends on the expertise of the examiner and the subjective report of the patient. Second, because $\mathrm{CN}$ involvement may be asymptomatic, it is difficult to diagnose $\mathrm{CN}$ palsy at an early stage by clinical neurological evaluation. In addition, neurological evaluation is not an optimal diagnostic method for assessing lesion localization and extension, which are critical factors in planning treatment, particularly in delineation of target volume.

With the excellent soft tissue contrast resolution and multiplanar imaging capability of MRI, direct visualization of smaller nerves and nerve branches is possible, making MRI a valuable tool in detecting and defining the extent of CN involvement in NPC. The difference and relationship between MRI findings of $\mathrm{CN}$ involvement and the symptoms and signs of $\mathrm{CN}$ dysfunction have not been fully addressed.

The goal of this study was to detect the difference and relationship between MRI findings suggestive of $\mathrm{CN}$ involvement and the symptoms and signs of $\mathrm{CN}$ dysfunction, and to evaluate the prognostic value of MRI-detected $\mathrm{CN}$ involvement in a large sample of consecutive patients. These information may contribute to understanding the patterns of spread and biological nature of NPC, and may also prove references in tumor staging and treatment planning.

\section{Patients and methods}

Patients: From January 2003 to December 2004, 924 consecutive patients with newly diagnosed untreated NPC were included in our study. There were 685 male patients and 239 female patients, with a male-female ratio of 2.9:1, and the median age was 45 years (range, 11-78 years). All patients had a pretreatment evaluation that consisted of a 
complete history, physical and neurologic examination, hematology and biochemistry profiles, MRI scan of the neck and nasopharynx, chest radiography, and abdominal sonography. Medical records and imaging studies were reviewed retrospectively, and all patients were staged according to criteria in the 6th edition of the AJCC Cancer Staging Manua.

Imaging Protocol: MR scanning was performed with a 1.5-Tesla system (Signa $\mathrm{CV} / \mathrm{i}$, General Electric Healthcare) and a head and neck combined coil. The area from the suprasellar cistern to the inferior margin of the sternal end of the clavicle was examined. T1weighted fast spin-echo images in the axial, coronal, and sagittal planes (repetition time of 500-600 milliseconds, echo time of 10-20 milliseconds, 5-mm slice thickness with 1-mm interslice gap for the axial plane, 6-mm slice thickness with 1-mm interslice gap for coronal and sagittal planes, and a 512*512 matrix) and T2-weighted fast spinecho MR images in the axial plane (repetition time of 4000-6000 milliseconds, echo time of 95-110 milliseconds, 5mm slice thickness with 1-mm interslice gap, and a 512*512 matrix) were obtained before injection of contrast agent. After intravenous gadolinium diethylenetriamine pentaacetic acid (Gd-DTPA; Magnevist, Schering, Berlin) injection at a dose of $0.1 \mathrm{mmol} / \mathrm{kg}$ of body weight, spin-echo T1-weighted axial and sagittal sequences, and spin-echo T1- weighted fatsuppressed coronal sequences were performed sequentially, with parameters similar to those used before Gd-DTPA injection.

\subsection{Image assessment and diagnostic criteria}

Medical records and imaging studies were retrospectively analyzed. Cranial nerve palsy was diagnosed separately by two radiation oncologists who were unaware of the clinical findings, and clinical nerve palsy was diagnosed by others on the basis of clinical symptoms and a physical examination before treatment. Any disagreements were resolved by consensus. Two radiologists specializing in head and neck cancers, who were unaware of the clinical findings, evaluated the MRI images separately. Any disagreements were resolved by consensus. Soft tissue tumor was observed as intermediate signal intensity on pre-Gd-DTPA T1- and T2-weighted images and an enhancing mass on post-Gd-DTPA T1weighted images, replacing the normal anatomy of the structure. MRI-detected CN involvement had to meet one of the following criteria: 1) enhancement of soft tissue tumor along the course of the ipsilateral related nerve and replacing the normal structures of the $\mathrm{CN}$ on gadolinium-enhanced T1-weighted images [Fig.1]; or 2) perineural spread, defined as an enlargement or abnormal enhancement of the nerve, obliteration of the neural fat pads adjacent to the neurovascular foramina, and neuroforaminal enlargement [Fig.1] [1-3]. Because $\mathrm{CNs}$ in the parapharyngeal space could not be identified as isolated structures on MRI, parapharyngeal space invasion could not be regarded as evidence of $\mathrm{CN}$ involvement in our study.

MRI-detected $\mathrm{CN}$ involvement at the following sites were assessed: extracranial segment of the maxillary nerve (V3), pterygopalatine fossa, foramen rotundum, foramen ovale, jugular foramen, hypoglossal canal, inferior orbital fissure, orbital apex, superior orbital fissure, cavernous sinus segment of CNs III-VI, trigeminal ganglion, and CNs in the cistern. By AJCC criteria, parapharyngeal and skull base invasion were classified as T2 and T3, respectively, and intracranial, infratemporal, hypopharynx, or orbital disease was classified 
as T4 [4]. Thus, all MRI findings that were suggestive of $\mathrm{CN}$ involvement were classified into 2 categories: 1) extracranial or basicranial or 2) intracranial or orbital.

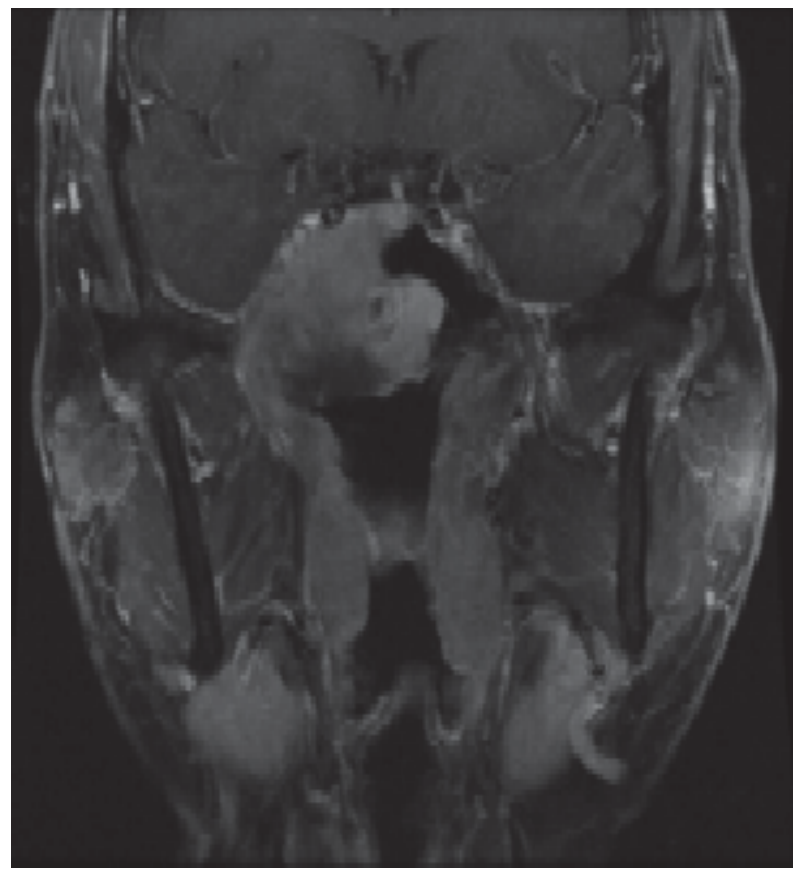

Fig. 1. A palsied cranial nerve V3. The enhanced coronal T1-weighted coronal fat suppression image at the level of the foramen ovale shows the enhancing tumor extending intracranially via the enlarged right foramen ovale along V3 and into the trigeminal cistern.

\subsection{Treatment}

All patients were treated by definitive-intent radiation therapy. Our policy was to cover the nasopharynx and the retropharyngeal lymph nodes within the primary target in every radical attempt, and to treat patients with gross lymphadenopathy with whole-neck irradiation. Most patients (773 of 924 or $83.7 \%$ ) were treated with conventional techniques, $12.7 \%$ (118 of 924) with intensity-modulated radiation therapy (IMRT), and 3.6\% (33 of 924) with 3-dimensional conformal radiation therapy (3-DCRT). Details regarding the radiation therapy techniques at the Cancer Center of Sun Yat-sen University have been reported previously [5-7].

Most patients (517 of 629 or $82.2 \%$ ) with stage III or stage IV disease (classified as T3-T4 or N2-N3) received neoadjuvant (137 of 629 or $21.8 \%$ ), concomitant (374 of 629 or $59.5 \%$ ), or adjuvant chemotherapy (6 of 629 or $1.0 \%$ ), in conjunction with a platinum-based therapeutic clinical trial. When possible, salvage treatments (including afterloading, surgery, and chemotherapy) were provided in the event of documented relapse or when the disease persisted despite therapy. 


\subsection{Statistical analysis}

Patients were followed up at least every 3 months during the first 2 years; thereafter, patients were followed up every 5 months until death. The median follow-up period for the whole group was 40 months (range, 2-56 months). All events were measured from the date of commencement of the treatment. The following endpoints (time to the first defining event) were assessed: overall survival (OS); local relapse-free survival (LRFS); and distant metastasis-free survival (DMFS). Local recurrence was established by fiberoptic endoscopy and biopsy and/or MRI. Distant metastases were diagnosed based on clinical symptoms, physical examination, and imaging methods including chest radiograph, bone scan, CT, and abdominal sonography.

Statistical analyses were performed using the Statistical Package for the Social Sciences (SPSS Inc., Chicago, IL) 12.0 software. The actuarial rates were calculated by the KaplanMeier method [8], and the differences were compared by using the log-rank test. Multivariate analyses with the Cox proportional hazards model were used to test independent significance by backward elimination of insignificant explanatory variables [9]. Host factors (age and sex) were included as the covariates in all tests. The chi-square test was used to analyze the relationship between MRI findings and $\mathrm{CN}$ palsies. The criterion for statistical significance was set at $\mathrm{a}=0.05$. The $P$ values were based on 2 -sided tests.

\section{Results}

\subsection{Clinical CN palsies and MRI-detected CN Involvement}

Of 924 patients with NPC, $82(8.9 \%)$ patients initially presented with CN palsy. A total of $134 \mathrm{CNs}$ were found to be paralyzed, and 31 patients were detected with multiple $\mathrm{CN}$ palsies. In these 82 patients with $\mathrm{CN}$ palsy, 79 patients had one or more MRI findings demonstrating $\mathrm{CN}$ involvement, and $\mathrm{CN} \mathrm{V}$ was the most common nerve to be involved. Of all patients, $46(5.0 \%)$ had clinical evidence of trigeminal nerve palsy at presentation, 92 (28\%) divisions of palsied trigeminal nerves were clinically identified. Of these 46 patients, $43(93.5 \%)$ had unilateral paresthesia and $3(6.5 \%)$ had bilateral paresthesia. In 5 of these patients with palsied nerves, MRI findings did not correspond to clinical findings. For example, a patient was detected with palsied CN V3, but MRI demonstrated only evidence of $\mathrm{CN}$ involvement of CN V2.

A total of $333(36 \%)$ patients demonstrated MRI-detected CN involvement and of the 514 patients with local advanced (T3-4) disease, 332 patients demonstrated MRI-detected CN involvement. The incidence of MRI-detected $\mathrm{CN}$ involvement in T3 patients was lower than that in T4 patients $(43.8 \%$ vs $97.5 \%, P=0.000)$. Only 1 patient with $\mathrm{T} 2$ disease demonstrated MRI-detected $\mathrm{CN}$ involvement. Clinical signs and symptoms of ipsilaterally affected CNs were absent in 259 (77.8\%) of patients with MRI-detected CN involvement. The correlation between MRI-detected CN involvement and ipsilateral CN palsy is shown in Table 1.

\subsection{Imaging basis of $\mathrm{CN}$ palsies}

Of the 134 paralyzed CNs, 98 (73.1\%) demonstrated ipsilateral intracranial or orbital MRIdetected CN involvement; 21 (15.7\%) demonstrated basicranial MRI-detected CN involvement. No MRI evidence of $\mathrm{CN}$ involvement was observed in the remaining 15 
(11.2\%) paralyzed CNs; among these $15 \mathrm{CNs}$, carotid sheath $(\mathrm{n}=13)$ or retropharyngeal lymph node $(n=1)$ invasion could be identified along the course of the CNs paralyzed on the ipsilateral side; no tumor could be detected along the course of the remaining paralyzed CNs on the MRI images. Of the 92 divisions of palsied trigeminal nerves, 91 trigeminal nerve palsies $(98.9 \%)$ showed MRI evidence of 1 or more trigeminal nerve involvements. However, no evidence could be found for the remaining 1 palsied V1 case on MR images.

\begin{tabular}{lcc}
\hline MRI-detecded CN involvement & Sites number* & Palsied CNs, Sites number \\
\hline Basicranial or extracranial & 264 & V3, 28 \\
Extracranial part of V3 & 223 & V3, 28 \\
Ovale foramen & 177 & V2, 30 \\
Pterygopalatine fossa & 86 & V2, 19 \\
Rotundum foramen & 48 & IX, 1; X, 2; XI, 0 \\
Jugular foramen & 108 & XII, 11 \\
Hypoglossal foramen & & \\
Intracranial or orbital & 6 & $\mathrm{III}, 1 ; \mathrm{IV}, 2 ; \mathrm{V} 1,2 ; \mathrm{VI}, 1$ \\
Superior orbital fissure & 11 & $\mathrm{II}, 5$ \\
Orbital apex & 36 & $\mathrm{~V} 2,14$ \\
Inferior orbital fissure & 41 & $\mathrm{III}, 10$ \\
Cavernous segment of III & 75 & $\mathrm{IV}, 11$ \\
Cavernous segment of IV & 130 & $\mathrm{~V} 1,20$ \\
Cavernous segment of V1 & 144 & $\mathrm{~V} 2,31$ \\
Cavernous segment of V2 & 125 & $\mathrm{VI}, 30$ \\
Cavernous segment of VI & 86 & $\mathrm{~V}, 24$ \\
Trigeminal ganglion & 9 & $\mathrm{~V}, 5$ \\
Cisternal part of V CN & 4 & $\mathrm{IX}-\mathrm{XI}, 0$ \\
Cisternal part of IX-XI & 9 & XII, 2 \\
Cisternal part of XII &
\end{tabular}

*: Sites number means the number of MRI-detected CN involvement

Table 1. The Correlation between MRI-detected CN Involvement and the Ipsilateral Related CN Palsy.

\subsection{Prognosis of MRI-detected CN Involvement in T3-4 disease}

In 514 T3-4 patients, significant differences were observed in 3-year OS (75.7\% vs $89.2 \%$, $P=0.001)$ and DMFS $(77.1 \%$ vs $87.8 \%, P=0.002)$ rates, with better rates observed in the 182 patients who did not demonstrate MRI-detected $\mathrm{CN}$ involvement. No significant difference was observed in 3-year LRFS ( $86.8 \%$ vs $91.8 \%, P=0.067)$ rates between the patients with and without MRI-detected CN involvement in T3-4 patients.

Because most (97.5\%) T4 patients had MRI-detected $\mathrm{CN}$ involvement, the prognoses between the T3 patients with and without MRI-detected $\mathrm{CN}$ involvement were also compared. All patients with local advanced disease (T3 and T4) in this series were classified into 3 groups: Group 1, T3 disease without MRI-detected CN involvement; Group 2, T3 disease with MRI-detected CN involvement; and Group 3, T4 disease. Significant differences were observed in OS and DMFS rates between Groups 1 and $2(P=0.009$ and $P=0.011$, 
respectively), but no significant differences were observed between Groups 2 and 3 $(P=0.322$ and $P=0.809$, respectively). No significant differences were observed in the LRFS rates between Groups 1 and $2(P=0.750)$, and no significant difference was observed between Groups 2 and $3(P=0.079)$.

Multivariate analysis was performed to adjust for various prognostic factors in T3 and T4 disease. Parameters were included in the Cox proportional hazards model by backward elimination of insignificant explanatory variables: age ( $50<=$ years vs $>50$ years), sex, skull base extension, paranasal sinus extension, intracranial extension, infratemporal extension, orbital extension hypopharynx extension, MRI-detected CN involvement, CN palsy, N classification, radiotherapy technique, and chemotherapy. Both clinical CN palsy and MRIdetected $\mathrm{CN}$ involvement were significant predictive factors for the DMFS and OS rates in local advanced disease (Table 2).

\begin{tabular}{llcccc}
\hline \multirow{2}{*}{ Endpoint } & Variable & $\mathrm{B}$ & $\operatorname{Exp}(\mathrm{B})$ & $\begin{array}{c}95 \% \mathrm{CI} \text { for } \\
\operatorname{Exp}(\mathrm{B})\end{array}$ & $P$ \\
\hline \multirow{4}{*}{ OS } & age & -0.287 & 0.750 & $0.550-1.024$ & 0.070 \\
& MRI-detected CN involvement & 0.866 & 2.377 & $1.620-3.489$ & 0.000 \\
& CN palsy & -0.662 & 0.516 & $0.300-0.886$ & 0.016 \\
& intracranial extension & 0.511 & 1.667 & $1.104-2.516$ & 0.015 \\
& N classification & 0.487 & 1.627 & $1.396-1.898$ & 0.000 \\
\multirow{5}{*}{ DMFS } & MRI-detected CN involvement & 0.627 & 1.871 & $1.268-2.762$ & 0.002 \\
& CN palsy & -0.506 & 0.603 & $0.330-1.102$ & 0.100 \\
& paranasal sinuses extension & 0.509 & 1.665 & $1.087-2.547$ & 0.019 \\
& N classification & 0.576 & 1.778 & $1.510-2.094$ & 0.000 \\
LRFS & age & -0.379 & 0.685 & $0.457-1.026$ & 0.066 \\
& gender & -0.855 & 0.425 & $0.232-0.779$ & 0.006 \\
& intracranial extension & 0.787 & 2.197 & $1.424-3.389$ & 0.000 \\
\hline
\end{tabular}

OS, overall survival; LRFS, local relapse-free survival; DMFS, metastasis-free survival.

Table 2. Summary of Multivariate Analyses of Prognostic Factors in T3-4 Disease.

\subsection{Difference in the prognostic implications between clinical $\mathrm{CN}$ palsy and MRI-detected CN involvement}

In 336 patients with clinical and/or MRI-detected CN involvement, no significant difference was observed in the 3 -year DMFS $(74.6 \%$ vs $84.6 \%, P=0.094)$ or LRFS $(86.7 \%$ vs $87.9 \%, P=$ $0.899)$ rates between patients with and without clinically detected $\mathrm{CN}$ palsies. A marginally significant difference was observed in the 3 -year OS $(74.2 \%$ vs $80.1 \%, P=0.067)$ rate; however, after adjusting for $\mathrm{N}$ classification, there was no significant difference $(P=0.102)$.

\subsection{Prognosis of lesion localization in patients with MRI-detected $\mathrm{CN}$ involvement}

Of the 333 patients with MRI-detected $\mathrm{CN}$ involvement, no significant differences were observed with regard to the 3 -year OS $(78.3 \%$ vs $72.9 \%, P=0.120)$, LRFS $(89.7 \%$ vs $84.1 \%$, $P=0.154)$ or DMFS $(79.6 \%$ vs $74.8 \%, P=0.466)$ rates between patients with and without intracranial or orbital MRI-detected $\mathrm{CN}$ involvement. 


\section{Discussion}

\subsection{Difference between clinical $\mathrm{CN}$ palsy and MRI-detected $\mathrm{CN}$ involvement}

Clinical findings were not consistent with the MRI findings of $\mathrm{CN}$ involvement in some cases. Nerves are resistant to tumor, and perineural tumor spread is an insidious and often asymptomatic process. The incidence of MRI-detected CN involvement is high in NPC; patients are often asymptomatic [10]. According to our observation, the incidence of MRIdetected $\mathrm{CN}$ involvement was much higher than that of clinical presentation of $\mathrm{CN}$ palsy in NPC patients.

With improvement in imaging techniques, direct visualization of smaller nerves and proximal nerve branches have become possible. MRI allows contrast between the nerve fibers and the surrounding cerebrospinal fluid, perineural vascular plexus, and fat pads. Involvement of cranial nerves may be direct or perineural; proposed MRI criteria of cranial nerve involvement in our study include both possibilities. MRI, including the contrastenhanced fat suppression technique, is particularly helpful in detection of direct and perineural invasion of the nerve in patients with NPC, so we often identified cranial nerve involvement on gadoliniumenhanced T1-weighted images. Perineural spread, in which pathologic conditions spread along the connective tissues of the perineurium, is often associated with malignant disease of the head and neck. The imaging hallmark of this process has been discussed in detail in a previous articles [1-3].

Without contrast between the nerve and the surrounding structures, CNs in the parapharyngeal space could not be identified on MRI; therefore, parapharyngeal space invasion was not defined as evidence of $\mathrm{CN}$ involvement in our study. A small number of symptomatic patients with only carotid sheath or retropharyngeal lymph node invasion along the course of ipsilaterally paralyzed CNs were regarded as MRI negative but clinically positive in our series.

Because NPC is a nonsurgical disease, the limitation of our study is that there was no pathologic correlation of $\mathrm{CN}$ involvement with MRI findings. Another limitation is the slice thickness of $5 \mathrm{~mm}$ used in our study, which may limit the sensitivity of detecting cranial nerve involvement. Ideally, slice thickness should be 3 to $4 \mathrm{~mm}$ in future studies; this may lead to earlier identification of $\mathrm{CN}$ involvement.

\subsection{Prognosis of clinical CN palsy in MRI-detected CN involvement}

We observed that most $\mathrm{CN}$ palsies had 1 or more MRI findings of intracranial or orbital CN involvement; this may be a reason for the adverse prognostic significance of $\mathrm{CN}$ palsies, and patients with $\mathrm{CN}$ palsies are determined to be at stage T4 [4].

Several investigators have reported that the perineural tumor spread in patients with nonnasopharyngeal carcinoma of the head and neck is associated with an increased incidence of recurrence [11-14]. However, in our series, MRI-detected CN involvement was not associated with the 3-year LRFS rate in NPC patients with local advanced disease. The primary treatment modality for NPC was radiation therapy, whereas that of other carcinomas of the head and neck was surgery. Lawrence and Cottel reported that postoperative radiotherapy of squamous cell carcinoma with perineural invasion resulted in a much improved survival probability when compared with that observed after 
conventional surgical excision [13]. Radiation therapy (relatively large treatment volume) provided better LRFS to patients with perineural spread. Early results of improved treatment strategies for nasopharyngeal carcinoma, including the boost technique of 2dimensional radiation therapy, use of IMRT, and combinationof chemotherapy with radiotherapy, has shown considerable improvement in LRFS [15-16]. Improved treatment might result in no significant difference with regard to the 3-year LRFS rate in patients with T3-4 disease with or without MRI findings of CN involvement.

The LRFS in patients with intracranial or orbital $\mathrm{CN}$ involvement was so poor that there was little difference in the 3-year LRFS rate between T3 patients with MRI-detected CN involvement and T4 patients. This poor survival rate may be a result of difficulties encountered in designing a therapeutic strategy for these patients and limitations on the dose escalation for intracranial or orbital disease. Several studies have demonstrated that perineural tumor spread in other head and neck cancers may result in a higher incidence of distant metastases [17-18]. We found that MRI-detected CN involvement was associated with a greater propensity for distant metastasis and resulted in unfavorable outcome in OS. Batsakis reported that carcinoma proliferated along the nerves within the lymphatics of the epineurium and the perineural sheaths [19]. We presume that tumor proliferation within the lymphatics may increase the risk of distant metastasis. On the basis of our data, the pattern of treatment failure in the NPC patients with $\mathrm{CN}$ involvement is caused by distant metastases. This implies that tumors with perineural involvement are more aggressive and spread from the primary tumor site to distant organs. Understanding the perineural invasion at the molecular level is an important step toward identification of the prognostic markers and therapeutic targets for NPC treatment.

Both clinical CN palsy and MRI-detected CN involvement were significant and independent predictive factors for the DMFS and OS rates in local advanced disease. In patients with clinical CN palsy or MRI-detected CN involvement, there is no real difference in the OS and DMFS between symptomatic and asymptomatic patients. This implies that MRI-detected involvement of CN itself has a poor prognosis in high-risk NPC irrespective of the clinical symptoms caused by such an involvement. In current AJCC Cancer Staging Manual, that CN involvement be assessed by neurological evaluation is recommended, rather than by crosssectional imaging. Therefore, MRI-detected $\mathrm{CN}$ involvement should be involved in the future staging system.

\subsection{Clinical value of MRI-detected CN involvement in treatment}

Almost all the patients with MRI-detected CN involvement were classified as T3-4. Concurrent chemoradiotherapy has emerged as the treatment of choice for locoregionally advanced (T3-4) NPC. Therefore, all patients with MRI-detected CN involvement should receive concurrent chemoradiotherapy. According to our data, these patients had a high distant metastasis rate; therefore, more intensive chemotherapy regimes should be considered.

Chang et al demonstrated that MRI was associated with improved tumor control of patients with NPC and CN palsy [20]. Because MRI can be used to clearly detect the location and extent of $\mathrm{CN}$ invasion, MRI should be considered as a basic imaging modality in the delineation of the target volume, ensuring full coverage of the gross target target volume 
and high-risk clinical target volume. A higher radiation dose improved tumor control and survival in NPC. We suggest that an adequate dose should be determined for these patients with MRI-detected CN involvement.

\subsection{Limitations of our study}

Prognosis may depend not only on the extent of disease on MRI but also on the effectiveness of treatment. Variability of treatment may be one of the limitations of our study. In our series, most patients with stage III and IV disease received chemotherapy, but some did not receive chemotherapy (patients with advanced age, heart disease, diabetes, and patients in the control group in a clinical trial). Although 3-DCRT/IMRT techniques were reported to provide excellent locoregional control for NPC, distant metastases are still the main cause of treatment failure [21]. In our series, only a small percentage of patients were treated with IMRT/3-DCRT because of resource limitations; however, when included as a covariate, radiation technique was not an independent prognostic factor for either distant failure or death in multivariate analyses.

\section{Summary}

The incidence of MRI-detected CN involvement in NPC is high and is often observed in asymptomatic patients, and the incidence of MRI-demonstrable trigeminal nerve involvement is much higher than other nevers. Disease with MRI-detected CN involvement has a poor prognosis, independent of lesion localization and symptoms in local advanced disease.

\section{References}

[1] Williams LS, Mancuso AA, Mendenhall WM. Perineuralspread of cutaneous squamous and basal cell carcinoma: CT and MR detection and its impact on patient management and prognosis. Int J Radiat Oncol Biol Phys. 2001;49:1061-1069.

[2] Gebarski SS, Telian SA, Niparko JK. Enhancement along the normal facial nerve in the facial canal: MR imaging and anatomic correlation. Radiology. 1992;183:391-394.

[3] Curtin HD, Wolfe P, Snyderman N. The facial nerve between the stylomastoid foramen and the parotid: computed tomographic imaging. Radiology. 1983;149:165-169.

[4] Lin JC, Jan JS. Locally advanced nasopharyngeal cancer: long-term outcomes of radiation therapy. Radiology. 1999;211:513-518.

[5] Jun Ma, Lizhi Liu, Linglong Tang, et al. Retropharyngeal lymphadenopathy in nasopharyngeal carcinoma: prognostic value and staging categories. Clin Cancer Res. 2007;13: 1445-1452.

[6] Zhao C, Han F, Lu LX, et al. Intensity modulated radiotherapy for local-regional advanced nasopharyngeal carcinoma. Ai Zheng. 2004;23:1532-1537.

[7] Wei L, Xiaowu D, Taixiang L. Dosimetric evaluation for 3 dimensional radiotherapy plans for patients with early nasopharyngeal carcinoma. Ai Zheng. 2004;23:605-608.

[8] Kaplan EL, Meier P. Nonparametric estimation from incomplete observations. J Am Stat Assoc. 1958;53:457-481.

[9] Cox DR. Regression models and life tables. J R Stat Soc B. 1972;34:187-220. 
[10] Neel HB. Nasopharyngeal carcinoma: clinical presentation, diagnosis, treatment and prognosis. Otolaryngol Clin North Am. 1985;18:479-490.

[11] Feasel AM, Brown TJ, Bogle MA, Tschen JA, Nelson BR. Perineural invasion of cutaneous malignancies. Dermatol Surg. 2001;27:531-542.

[12] Fagan JJ, Collins B, Barnes L, D'Amico F, Myers EN, Johnson JT. Perineural invasion in squamous cell carcinoma of the head and neck. Arch Otolaryngol Head Neck Surg. 1998;124:637-640.

[13] Lawrence N, Cottel WI. Squamous cell carcinoma of skin with perineural invasion. J Am Acad Dermatol. 1994;31:30- 33.

[14] McCord MW, Mendenhall WM, Parsons JT, Flowers FP. Skin cancer of the head and neck with incidental microscopic perineural invasion. Int J Radiat Oncol Biol Phys. 1999;43:591-595.

[15] Xie GF, Cao KJ, Li Y, Huang PY. Impact of dose boost in skull base on recurrence of stage T4 nasopharyngeal carcinoma. Ai Zheng. 2005;24:1246-1248.

[16] Ma J, Mai HQ, Hong MH, et al. Results of a prospective randomized trial comparing neoadjuvant chemotherapy plus radiotherapy with radiotherapy alone in patients with locoregionally advanced nasopharyngeal carcinoma. J Clin Oncol. 2001;19:1350-1357.

[17] Ballantyne AJ, McCarten AB, Ibanez ML. The extension of cancer of the head and neck through peripheral nerves. Am J Surg. 1963;106:651-667.

[18] Byers RM, O'Brien J, Waxler J. The therapeutic and prognostic implications of nerve invasion in cancer of the lower lip. Int J Radiat Oncol Biol Phys. 1978;4:215-217.

[19] Batsakis JG. Nerves and neurotropic carcinomas. Ann Otol Rhinol Laryngol. 1985;94:426-427.

[20] Chang JT, Lin CY, Chen TM, et al. Nasopharyngeal carcinoma with cranial nerve palsy: the importance of MRI for radiotherapy. Int J Radiat Oncol Biol Phys. 2005;63:13541360.

[21] Kam MK, Teo PM, Chau RM, et al. Treatment of nasopharyngeal carcinoma with intensity modulated radiotherapy: the Hong Kong experience. Int J Radiat Oncol Biol Phys. 2004;60:1440-1450. 


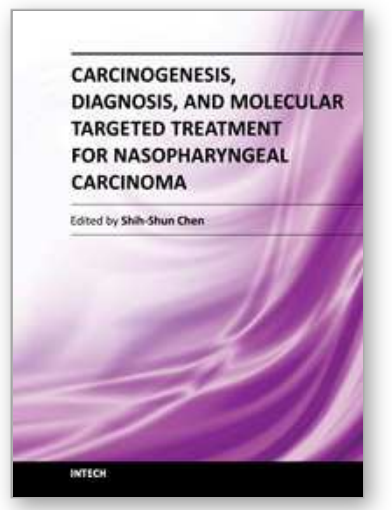

\section{Carcinogenesis, Diagnosis, and Molecular Targeted Treatment for Nasopharyngeal Carcinoma}

Edited by Dr. Shih-Shun Chen

ISBN 978-953-307-867-0

Hard cover, 246 pages

Publisher InTech

Published online 15, February, 2012

Published in print edition February, 2012

This book is a comprehensive treatise of the potential risk factors associated with NPC development, the tools employed in the diagnosis and detection of NPC, the concepts behind NPC patients who develop neuroendocrine abnormalities and ear-related complications after radiotherapy and chemotherapy, the molecular mechanisms leading to NPC carcinogenesis, and the potential therapeutic molecular targets for NPC.

\section{How to reference}

In order to correctly reference this scholarly work, feel free to copy and paste the following:

Li Li, Wenxin Yuan, Lizhi Liu and Chunyan Cui (2012). MRI-Detected Cranial Nerve Involvement in Nasopharyngeal Carcinoma, Carcinogenesis, Diagnosis, and Molecular Targeted Treatment for Nasopharyngeal Carcinoma, Dr. Shih-Shun Chen (Ed.), ISBN: 978-953-307-867-0, InTech, Available from: http://www.intechopen.com/books/carcinogenesis-diagnosis-and-molecular-targeted-treatment-fornasopharyngeal-carcinoma/mri-detected-cranial-nerve-involvement-in-nasopharyngeal-carcinoma

\section{INTECH}

open science | open minds

\author{
InTech Europe \\ University Campus STeP Ri \\ Slavka Krautzeka 83/A \\ 51000 Rijeka, Croatia \\ Phone: +385 (51) 770447 \\ Fax: +385 (51) 686166 \\ www.intechopen.com
}

\author{
InTech China \\ Unit 405, Office Block, Hotel Equatorial Shanghai \\ No.65, Yan An Road (West), Shanghai, 200040, China \\ 中国上海市延安西路65号上海国际贵都大饭店办公楼 405 单元 \\ Phone: +86-21-62489820 \\ Fax: $+86-21-62489821$
}


(C) 2012 The Author(s). Licensee IntechOpen. This is an open access article distributed under the terms of the Creative Commons Attribution 3.0 License, which permits unrestricted use, distribution, and reproduction in any medium, provided the original work is properly cited. 